\title{
Service user perspectives on coercion and restraint in mental health
}

\author{
Diana Rose, ${ }^{1}$ Emma Perry, ${ }^{2}$ Sarah Rae ${ }^{3}$ and Naomi Good ${ }^{4}$
}

2Professor of User-Led Research and Co-director of Service User Research Enterprise (SURE),

King's College London, UK; email diana.rose@kcl.ac.uk ${ }^{2}$ Research Project Coordinator, National Survivor User Network (NSUN), UK

${ }^{3}$ Cambridgeshire and

Peterborough NHS Foundation

Trust (CPNHSFT) and joint lead

the Promise initiative, UK

${ }^{4}$ Networking and Research

manager, National Survivor User Network (NSUN)

Conflict of interest. No authors have any conflicts of interest to declare.

PSYCHIATRY UNDER RESTRICTIVE CONDITIONS
Coercion remains a central aspect of many people's mental healthcare. It can include the use of legislation to restrict freedoms, the use of physical restraint, the restriction of freedom of movement and/or association, and the forced or covert administration of medication. There is good evidence that the use of such measures can traumatise service users. This article reports the findings of a survey of service users regarding their experiences of coercion and restraint and embeds this in the wider international and institutional environment.

Most jurisdictions in the industrialised West promulgate mental health legislation which permits infringement of liberty and treatment against the will of the person detained. Increasingly, coercion in the community is also allowed. Partly at the behest of the World Health Organization, other countries are developing similar legislation. This ability to compel and coerce is unique in medicine and, from within psychiatry, has been called discriminatory (Dawson \& Szmukler, 2006) and criticised for the focus on risk.

Dealing first with the United Nations' 2006 Convention on the Rights of Persons with Disabilities (CRPD) and its implications for coercion and compulsion globally, this article summarises what is known about service users' responses to coercion and compulsion, which includes chemical, physical and mechanical restraint. Responding to the paucity of studies focusing specifically on physical restraint (which is commonly followed by chemical restraint), this article reports on recent experiences of people subject to this practice in the UK while situating this in more general concerns.

\section{The CRPD}

The CRPD appears to be top of the agenda for service user movements everywhere except the UK. The European Network for (ex) Users and Survivors of Psychiatry (ENUSP) has provided a point-by-point explanation of how European human rights legislation is in breach of the CRPD and the World Network (WNUSP) was involved in drawing up the Convention itself. It is important as it is the first time that people "with psychosocial disabilities' have been recognised as equal in law to other citizens in terms of rights.

But the Convention is controversial, particularly since the publication of the General Comment on Article 12, which states that all persons with disabilities must be deemed to have legal capacity all of the time. This intensifies the commitment in
Article 14 to abolish compulsory detention and treatment by doing away with the last criterion on which it could be justified. The General Comment has drawn the ire of leading Western psychiatrists (Freeman et al, 2015). The debate is also not settled within service user circles, as shown by the different positions of Tina Minkowitz and Anne Plumb (see Spandler et al, 2015).

\section{Global South}

The CRPD language of "persons with psychosocial disabilities' has also had important implications in the Global South. There is a move from the terms 'user/survivor' to 'persons with psychosocial disabilities'. For example, the Pan African Network of Users and Survivors of Psychiatry is now the Pan African Network of Persons with Psychosocial Disabilities. A similar move has been counselled in India (Davar, 2013). This is not simply a change in language but a political position that states 'we don't have psychiatry and don't want it'.

An Indonesian government programme, Aceh Free Pasung, intends to protect the human rights of people with severe mental illness by eliminating the practice of pasung from the province. Pasung means restraint by shackles, rope, wooden stocks and cages, and can cause death. Since the alternative was the building of a mental health hospital, the initiative has enabled access to free hospital treatment (Minas et al, 2011). Its replacement with neuroleptics, restraint and hospital confinement is nevertheless problematic.

\section{Industrialised West}

Decisions about coercion often hinge on the concept of risk. Recent evidence suggests that risk assessments exclude service users from the discussion and render invisible the fact that the assessments are more or less fictions in actuarial terms (Coffey et al, 2017).

How risk of harm within mental health settings is perceived and managed has received attention. However, harm in the context of restraint is mostly not recognised. Nonetheless, restraint-related deaths have been reported throughout the Western world, including in the UK, where 38 deaths have been recorded since 1988, 15 of people from Black and Minority Ethnic (BME) communities (Aiken et al, 2011). A qualitative study of in-patients showed that nurses and patients had very different accounts of both the determinants of and appropriate responses to violence and aggression (Rose et al, 2015).

Reviewing evidence of the effects of restraint, Cusack et al (2016) concluded that 'restraint can be 
a form of abuse, its inappropriate use often being a consequence of fear, neglect and lack of using deescalation techniques'.

A small pilot study by the National Survivor User Network (NSUN), as yet unpublished, surveyed 65 people about their experiences of restraint and recruited respondents mainly through the weekly e-bulletin. The findings align with Strout's (2010) integrative review of the international qualitative literature on patient perspectives on the use of restraint. Strout identifies four categories: negative psychological impact, re-traumatisation, perceptions of unethical practices, and the broken spirit. Of the respondents surveyed in the NSUN study, $43 \%(n=28)$ stated that their most recent experience of restraint had felt like an act of aggression 'a great deal', 12\% $(n=8)$ 'a lot' and 15\% $(n=10)$ 'somewhat'. Although the number was small, all respondents from BME communities $(n=5)$ stated that their experience had felt like an act of aggression 'a great deal' or 'a lot'. Respondents frequently cited the unnecessary use of physical force and the number of staff members who were involved in each incident of restraint. The most frequent reason for restraint given by respondents was the refusal to take medication, and physical restraint was routinely followed by a rapid tranquillising injection. This was often experienced as shaming and humiliating:

When I become agitated it is very obviously extreme fear and terror. On each occasion it is ALWAYS male staff pinning me down - always face down so [I] can't breathe or move and who pull my underwear off/down to inject. They know the abuse history - it has been a police case. They never have talked to me afterwards. It is like being raped again.

One clear theme was the link between instances of physical restraint and tranquillisation with retraumatisation. Consistent with studies of women's experience of restraint representing an event that re-enacts the experience of trauma and sexual abuse, Gallop et al (1999) found that restraint and associated feelings of humiliation, punishment and powerlessness engendered traumatic emotional reactions such as fear, anxiety and rage.

Half of the respondents stated that experiencing physical restraint had affected their engagement with services 'a great deal'. The majority reported a loss of trust in staff, and increased fear, suspicion and wariness. Others reported that they avoided services because of their experiences.

The Promise study (Wilson et al, 2015), which informs the development of a proactive care framework to reduce the need for physical intervention, involved interviews with both staff and patients. The most dominant theme to emerge centred on staff-patient communication and relationships, with 11 patients and 19 staff members providing suggestions:

if you wish to reduce the number of restraints a high level of communication is needed whatever the issues of a particular patient, whatever their predisposition to violence or hatred of psychiatric staff, or factions within the resident group, or dislikes of a particular patient. In an ideal world there must be more verbal communication ... the culture is to encourage what Winston Churchill used to say was a 'jaw jaw rather than war war', to counsel and support a person onto a different state of thinking. (Wilson et al, 2015)

\section{Conclusion: minimising coercion and restraint}

In the NSUN study, $94 \%(n=50)$ of respondents reported that restraint could have been managed very differently, emphasising that if staff had taken the time to listen and to speak to them addressing fears, frustrations and concerns, the situation could have been avoided. Respondents said that staff needed better training and that service users should be involved in this.

No differences were found in the experiences of restraint occurring within the past 10 years compared with the past 2 years. Of the NSUN respondents, $40 \%(n=23)$ reported incidents of restraint that occurred within the past 2 years, suggesting no recent improvement in experiences of restraint. These findings signal that the 2-year UK government programme 'Positive and Safe', launched in 2014 with the goal of reducing the need for physical restraint (Department of Health, 2014), has had no impact to date and that a fundamental culture change is needed. This directive was followed by an investigation involving the facedown restraint of a woman eight and a half months pregnant in July 2016 (Mental Health Today, 2016).

There are various arguments for reducing coercion and restraint and many different approaches that service users say they find more beneficial. Since 2007 the Mental Health Act in England has made it mandatory for advocacy to be practised by professionals alone, although the service user voice is not completely absent (Newbigging et al, 2015). It will be important to regain and strengthen the user voice within peer and self-advocacy, as has happened in Africa (Kleintjes et al, 2013).

Joint crisis plans or advance directives are counselled by many as a means of reducing incidents of restraint and of listening to the needs of service users (Papageorgiou et al, 2002; Amering et al, 2005). Approaches that include peer facilitators and improvements to the frequency and quality of communications are crucial. Some existing institutional systems make good communication a practical impossibility.

From the perspective of service users, coercion and restraint are mostly harmful and must stop being legitimised. There is an urgent need to challenge and address these practices as they represent gross human rights violations according to the stipulations of the CRPD. UK compliance with the legislation is due to be monitored in the next 2 years.

\section{References}

Aiken, F., Duxbury, J., Dale, C., et al (2011) Review of the Medical Theories and Research Relating to Restraint Related Deaths. Caring Solutions (UK) and the University of Central Lancashire.

Amering, M., Stastny, P. \& Hopper, K. (2005) Psychiatric advance directives: qualitative study of informed deliberations by mental health service users. British Journal of Psychiatry, 186, 247-252. 
Coffey, M., Cohen, R., Faulkner, A., et al (2017) Ordinary risks and accepted fictions: how contrasting and competing priorities work in risk assessment and mental health care planning. Health Expectations, 20, 471-483.

Cusack, P., McAndrews, S. \& Warne, T. (2016) Restraining good practice: reviewing evidence of the effects of restraint from the perspective of service users and mental health professionals in the United Kingdom (UK). International Journal of Law and Psychiatry, 46, 20-26.

Davar, B. (2013) From mental illness to disability: choices for women users/survivors of psychiatry in self and identity constructions. In Disability Studies in India: Global Discourses, Local Realities (ed. R. Addlakha), pp. 333-360. Routledge.

Dawson, J. \& Szmukler, G. (2006) Fusion of mental health and incapacity legislation. British Journal of Psychiatry, 188, 504-509.

Department of Health (2014) Positive and Proactive Care: Reducing the Need for Restrictive Interventions. Department of Health. Available at https://www.gov.uk/government/uploads/system/ uploads/attachment data/file/300293/JRA DoH Guidance on RP_web_accessible.pdf (accessed June 2017).

Freeman, M. C., Kolappa, K., de Almeida, J. M. C., et al (2015) Reversing hard won victories in the name of human rights: a critique of the General Comment on Article 12 of the UN Convention on the Rights of Persons with Disabilities. Lancet Psychiatry, 2, 844-850.

Gallop, R., McCay, E., Guha, M., et al (1999) The experience of hospitalization and restraint of women who have a history of childhood sexual abuse. Health Care for Women International, 20, 401-416.

Kleintjes, S., Lund, C. \& Swartz, L. (2013) Organising for selfadvocacy in mental health: experiences from seven African countries. African Journal of Psychiatry, 16, 187-195.
Mental Health Today (2016) Investigation launched after pregnant mental health patient 'pinned to floor'. Available at https://www. mentalhealthtoday.co.uk/investigation-launched-after-pregnantmental-health-patient-pinned-to-floor.aspx (accessed 25 August 2016).

Minas, H., Puteh, I. \& Marthoenis, M. (2011) Aceh Free Pasung: releasing the mentally ill from physical restraint. International Journal of Mental Health Systems, 5, 10.

Newbigging, K., Ridley, J., McKeown, M., et al (2015) 'When you haven 't got much of a voice': an evaluation of the quality of Independent Mental Health Advocate (IMHA) services in England. Health and Social Care in the Community, 23, 313-324.

Papageorgiou, A., King, M., Janmohamed, A., et al (2002) Advance directives for patients compulsorily admitted to hospital with serious mental illness. British Journal of Psychiatry, 181, 513-519.

Rose, D., Evans, J., Laker C., et al (2015) Life in acute mental health settings: experiences and perceptions of service users and nurses. Epidemiology and Psychiatric Sciences, 24, 90-96.

Spandler, H., Anderson, J. \& Sapey, B. (2015) Madness, Distress and the Politics of Disablement. Policy Press.

Strout, T. (2010) Perceptions on the experience of being physically restrained: an integrative review of the qualitative literature. International Journal of Mental Health Nursing, 19, 416-427.

Wilson, C., Rouse, L., Rae, S., et al (2015) Promise: an exploration of staff and patient experience of physical intervention on mental health wards and their suggestions for reducing its use. Available at http://www.promise.global/9pc_1_5 cw.html (accessed 2 September 2016).

\section{THEMATIC} PAPER

\title{
Key international themes in coercion
}

\author{
Andrew Molodynski, ${ }^{1}$ Anthony O'Brien ${ }^{2}$ and Jonathan Burns ${ }^{3}$
}

${ }^{1}$ Consultant Psychiatrist, Oxford Health NHS Foundation Trust: Honorary Senior Lecturer, University of Oxford; Chair, World Association of Social Psychiatry Working Group on Coercion; Working Group on Coercion;
email andrew.molodynski@ email andrew.molodys
oxfordhealth.nhs.uk

2Senior Lecturer in Mental Health Nursing, University of Auckland; Nurse Specialist in Liaison Psychiatry, Auckland City Hospital, New Zealand ${ }^{3}$ Professor of Psychiatry, Nelson Mandela School of Medicine University of KwaZulu-Natal, Durban, South Africa UNDER RESTRICTIVE CONDITIONS
Coercion remains a dominant theme in mental healthcare and a source of major concern. While the presence of coercion is ubiquitous internationally, it varies significantly in nature and degree in different countries and is influenced by a variety of factors. Recent reports have raised concerns about physical restraint and the increasing use of legislation in highincome countries. At the same time, a recent Human Rights Watch report on pasung (the practice of tying or restricting movement more generally) in Indonesia has served to highlight the plight of many in middle- and lower-income countries who are subject to degrading and dehumanising 'treatment'.

The containment and coercion of people who have a mental illness has always been at the heart of mental healthcare, and the subject of fierce debate. Concerns initially focused on conditions and restrictions of liberty in the earliest facilities, the so-called 'mad houses'. In many high-income group (HIG) countries, care for the mentally ill moved into large asylums in the 19th century and concerns shifted to the quality of care, legal rights and institutionalisation of residents. Most care in these countries is now delivered outside institutions and though concerns persist regarding in-patient care, there is also more focus on coercion in the community. Coercion can be formal (the use of legal sanctions) or informal (the use of 'softer', less easily measurable pressures to bring about compliance) (Szmukler \& Appelbaum, 2008). Coercion in institutions is often divided into categories: legal, chemical and/or physical restraint, and the restriction of movement and association. This brief overview will not consider medication, although the coerciveness of surreptitious medication should be noted, and is an area of particular concern in India (Rao et al, 2012).

In HIG countries there has been a move towards increased scrutiny of coercive measures in hospitals and an extension of legal powers and informal coercion outside them (Molodynski et al, 2016). In other countries the issues may be very different, as evidenced by the recent Human Rights Watch report on pasung (Human Rights Watch, 2016), which cited Indonesian government data that approximately 19000 people are 